\title{
Effects of preservative-free tafluprost on tear film osmolarity, tolerability, and intraocular pressure in previously treated patients with open-angle glaucoma
}

This article was published in the following Dove Press journal:

Clinical Ophthalmology

II January 2012

Number of times this article has been viewed

\section{Ingrida Janulevičienè \\ Irmante Derkač \\ Lina Grybauskiene \\ Ruta Paulauskaitè \\ Ruta Gromnickaite \\ Loreta Kuzmienè}

Eye Clinic of Kaunas Medical Academy of Lithuanian University of Health Sciences, Kaunas, Lithuania
Correspondence: Ingrida Janulevičienè Eye Clinic of Kaunas Medical Academy of Lithuanian University of Health Sciences, Eiveniu str 2, LT-50009

Kaunas, Lithuania

Tel +37037326558

$\mathrm{Fax}+37037326146$

Email ingrida.januleviciene@kaunoklinikos.lt
Purpose: To compare the effects on tolerability, tear osmolarity, and intraocular pressure (IOP)-lowering effect of switching from benzalkonium chloride (BAK) containing prostaglandin analog (PGA) latanoprost to preservative-free tafluprost.

Patients and methods: Thirty patients with open-angle glaucoma ( $\mathrm{N}=60$ eyes), 26 women (87\%) and four men (13\%) aged 64.1 (SD 14.1) years, showing abnormal values of tear osmolarity, corneal fluorescein staining, tear film break-up time (TBUT), or subjective discomfort with current latanoprost treatment were included. After tear osmolarity (TearLab ${ }^{\mathrm{TM}}$ Osmolarity System), TBUT, corneal fluorescein staining, and baseline IOP (Goldmann tonometer) measurements and the completion of Ocular Surface Disease Index and Ocular Surface Symptoms in Glaucoma Scale questionnaires, patients were assigned to preservative-free tafluprost treatment. Measurements were repeated 2, 6 and 12 weeks after change of medication.

Results: No statistically significant differences in IOP were observed 2, 6, and 12 weeks after switching to preservative-free tafluprost. Mean IOP at baseline was $16.4 \mathrm{mmHg}$ (SD 2.9), after 2 weeks $16.2 \mathrm{mmHg}$ (2.8), after 6 weeks 16.2 (2.6), and after 12 weeks $16.3 \mathrm{mmHg}(2.3)$. Mean tear osmolarity decreased significantly from $315.7 \mathrm{mOsm} / \mathrm{L}$ (SD 15.1) at baseline to $308.0 \pm 14.4 \mathrm{mOsm} / \mathrm{L}(P=0.002), 301.7 \pm 14.5 \mathrm{mOsm} / \mathrm{L}(P<0.001)$, and $302.0 \pm 9.9 \mathrm{mOsm} / \mathrm{L}$ $(P<0.001) 2,6$, and 12 weeks after changing medication to preservative-free tafluprost, respectively. Tear osmolarity was lower in 37 eyes $(61.7 \%)$ after 2 weeks, in 46 eyes $(76.7 \%)$ after 6 weeks, and in 49 eyes $(81.7 \%)$ after 12 weeks $(P<0.005 ; t$-test $)$. At baseline corneal fluorescein staining was observed in 43 eyes (71.7\%), after 2 weeks in 34 eyes (56.7\%), after 6 weeks in 12 eyes (20.0\%), and after 12 weeks in 7 eyes $(11.7 \%)(P<0.005$; McNemar test). Mean TBUT increased from 3.7 seconds (SD 1.1) at baseline to 4.1 seconds (SD 1.0) at week 2, 5.2 seconds (SD 1.5) at week 6, and 6.5 seconds (SD 1.5) at week 12 ( $P<0.001 ; t$-test). The number of patients expressing discomfort with latanoprost diminished from 30 (100\%) at baseline, to $19(63.3 \%)$ after week 2, and to $11(36.6 \%)(P<0.05$; McNemar test $)$ after 12 weeks.

Conclusion: Preservative-free tafluprost is better tolerated than BAK-containing latanoprost, showing lower tear osmolarity levels while maintaining effective IOP control.

Keywords: glaucoma, preservatives, tafluprost, osmolarity, intraocular pressure, prostaglandin analogs, BAK, tolerability

\section{Introduction}

Glaucoma is a chronic, potentially blinding condition and one of the leading causes of blindness in the world. Medical therapy is the initial option in the treatment of glaucoma. ${ }^{1}$ 
However, adverse effects associated with topical medication may have a negative effect on patient adherence to medical treatment, doctor-patient relationship, and patient quality of life. ${ }^{2}$ Currently available topical glaucoma medication in multidose bottles typically contain preservatives in addition to the active component. After instillation, eye drops interact with ocular surface tissues. ${ }^{3}$ This interaction can involve both the active agent and the preservatives. Preservatives in eyedrops are used to inhibit microbial growth in the bottle, which allows safe use of the multidose containers. Preservatives also prevent biodegradation and support the maintenance of drug potency. ${ }^{4}$ Benzalkonium chloride (BAK), the most widely used preservative in topical glaucoma medications, is a cationic surfactant that adheres to microorganism cell membranes, increases membrane permeability, and leads to cell lysis. ${ }^{5}$ It has been demonstrated in various studies that BAK has also a toxic effect on the ocular surface. This preservative decreases tear film stability and turnover, causes inflammatory cell infiltration, and induces conjunctival and corneal epithelial cell abnormalities. ${ }^{6}$ The tear film is a dynamic structure comprising of lipid, aqueous, and mucin layers. Its production and turnover is essential for maintaining the health of the ocular surface. The formation and stability of the tear film in both health and disease are dependent upon the physical properties of the tear fluid. The tear film plays an important role in nourishing, lubricating, and protecting the ocular surface. A dysfunction of any of the layers of the tear film may result in dry eye disease. ${ }^{7}$ Disruption of the homeostasis of the tear film results in ocular surface inflammation, which may lead to cell damage. Abnormalities of any tear component can result in tear film instability and hyperosmolarity. ${ }^{8}$ Osmolarity is the measure of solute concentration, defined as the number of osmoles of solute per liter of solution $(\mathrm{Osm} / \mathrm{L}){ }^{9,10}$ As a measure of tear film chemistry, osmolarity can be useful for evaluating the quality of patients' tears. Tear film osmolarity in normal volunteers seems to be in the range of 290-300 mOsm/L. Studies reveal an average osmolarity of around $302 \mathrm{mOsm} / \mathrm{L} \cdot{ }^{11-14}$ Considering that tears are physiologically derived from blood, normal tear osmolarity should be observed around $290 \mathrm{mOsm} / \mathrm{L}$. Osmolarity of tears specifically refers to the concentration of small proteins and electrolytes, including sodium, potassium, and chloride. Although measuring osmolarity does not reveal the exact chemical composition of tears, it quantifies the concentration of the different components. Research has shown that knowledge of tear film osmolarity can be clinically valuable for assessing dry eye disease. Human tears are isotonic with a $0.9 \%$ sodium chloride solution ${ }^{15,16}$ and the recommendation for adjusting the osmotic concentration of eye drops to this presumed tonicity has found general acceptance. ${ }^{10}$ Tear hyperosmolarity is regarded as a central mechanism causing dry eye symptoms and ocular surface inflammation by activating a cascade of inflammatory events and the release of inflammatory mediators into the tears. Epithelial damage involves cell death by apoptosis, a loss of goblet cells, and a reduction in mucus secretion that leads to tear film instability. ${ }^{17,18}$ This instability exacerbates ocular surface hyperosmolarity, thereby creating a vicious circle. ${ }^{19}$ Recently, Lemp reported that osmolarity is the best single metric both to diagnose and classify dry eye disease. ${ }^{19}$ Tafluprost is a novel prostaglandin analog (PGA) that has been approved for ophthalmic use in a number of markets worldwide. The drug is currently marketed under the brand names Taflotan and Taflotan sine (Santen Oy, Tampere, Finland), Tapros (Santen Pharmaceutical Co Ltd, Osaka, Japan), and Saflutan (Merck Sharp and Dohme, Whitehouse Station, NJ). Among all widely used PGA's, tafluprost is the first and only preparation available in a preservative-free formulation. The aim of the current study was to compare the effects on tolerability, tear osmolarity, and intraocular pressure (IOP) lowering after switching from BAKpreserved latanoprost to preservative-free PGA tafluprost.

\section{Material and methods Study design}

This was a prospective, observer-masked study over a period of 12 weeks. The study was conducted in accordance with the Declaration of Helsinki. All subjects read and signed an informed consent, and the study was approved by the Kaunas University of Medicine Institutional Review Board.

\section{Patients}

Thirty patients with open-angle glaucoma $(\mathrm{N}=60$ eyes $)$ were included in the study. Patients were aged at least 18 years and willing to sign the informed consent prior to initiation of study. Inclusion criteria were: clinical diagnosis of open-angle glaucoma (with or without pseudoexfoliation or pigment dispersion component) in at least one eye with best-corrected visual acuity 20/40 (or equivalent) or better, at least mild dry eye according to Ocular Surface Disease Index (OSDI) score (while score 0 corresponds to never had any ocular surface disease symptoms) and/or corneal fluorescein staining in at least one eye (mild staining, less than $10 \%$ coverage of corneal surface; moderate, $10 \%-50 \%$ of corneal surface; severe, more than $50 \%$ of corneal surface), IOP controlled with monotherapy using latanoprost $50 \mu \mathrm{g} / \mathrm{mL}$ for not less than 1 continuous month immediately prior to the study visit. 
Patients with any abnormality preventing reliable applanation tonometry in the study eye(s), having dry eye treated with the use of punctual plugs, punctual cautery, cyclosporine, topical ocular corticosteroids, using artificial tears not discontinued prior to first study visit, having keratorefractive ocular laser procedures, corneal surgery or surgery to the corneal surface within one year prior to first visit, patients who have undergone intraocular or extraocular surgery ocular laser surgery in either eye within 6 months, patients with progressive retinal or optic nerve disease, patients with severe central visual field loss, patients with any history of infectious or inflammatory ocular conditions, patients having ocular trauma within 6 months, any systemic medications on a chronic basis that have not been on a stable dosing regimen for 1 month prior to the study, and history of intolerance or hypersensitivity to any component of the test articles were excluded from the study.

\section{Measurements and statistical analysis}

After baseline IOP (Goldmann applanation tonometry) and tear osmolarity (TearLab ${ }^{\mathrm{TM}}$ Osmolarity System; TearLab, San Diego, CA) measurements, answering two questionnaires, OSDI and Ocular Surface Symptoms in Glaucoma Scale (OSSG), patients were switched to preservative-free tafluprost for 12 weeks. IOP and tear osmolarity were measured and corneal staining evaluated at 2, 6, and 12 weeks. All measurements were done at the same time of day (3 pm). At the final study visit (week 12) patients filled OSSG and OSDI questionnaires again.

Several hypotheses were tested: primary, tear osmolarity level is restored after switching to preservative-free glaucoma medication; secondary, patients prefer preservative-free medication; tertiary, no statistically significant difference in IOP level between BAK-containing and preservative-free prostaglandin analogs. The primary efficacy variable was tear film osmolarity level as measured by TearLab [Osm/L]. Secondary efficacy variables were the IOP-lowering effect, tear film break-up time (TBUT), OSSG, and OSDI.

Descriptive statistics were obtained for the resulting measurements. In the event that significance was achieved by repeated analysis of variance (ANOVA) measurements, the Fisher's and Bonferroni models were applied. Changes in individual parameters were examined by paired Student's $t$-test, considering values of $P<0.05$ as statistically significant. To test the hypothesis that the mean difference between two measurements is zero, Wilcoxon signed-rank test was used. Changes in various parameters were analyzed by Pearson's correlation analysis.

\section{Results}

\section{Patient demographics and diagnoses}

Thirty patients with open-angle glaucoma (60 eyes), 26 women $(86.7 \%)$, four men $(13.3 \%)$, aged 64.2 years (SD 13.9 years) (range, 35-79 years) were recruited (Table 1). After baseline measurements, patients were assigned to preservative-free tafluprost treatment and measurements were repeated 2, 6, and 12 weeks after switching to preservativefree tafluprost. At baseline all patients reported subjective discomfort with their current latanoprost treatment.

\section{Osmolarity}

Mean tear osmolarity level at baseline (BAK-preserved latanoprost) was $315.7 \mathrm{mOsm} / \mathrm{L}$ (SD: 15.1) (Figure 1). At baseline, osmolarity was not statistically significant between the right and left eyes of patients. Compared to baseline, tear osmolarity decreased in 37 eyes (61.7\%) after 2 weeks, in 46 eyes $(76.7 \%)$ after 6 weeks, and in 49 eyes $(81.7 \%)$ after 12 weeks $(P<0.005$; $t$-test for paired samples) (Figure 1$)$. Mean osmolarity decreased significantly 2,6 , and 12 weeks after changing medication to $308.0 \pm 14.4 \mathrm{mOsm} / \mathrm{L}(P=0.002$ vs baseline), $301.7 \pm 14.5 \mathrm{mOsm} / \mathrm{L}(P<0.001 \mathrm{vs}$ baseline $)$, and $302.0 \pm 9.9 \mathrm{mOsm} / \mathrm{L}(P<0.001)$, respectively (Figure 2$)$.

\section{TBUT and fluorescein staining}

Mean TBUT $( \pm \mathrm{SD})$ increased significantly from $3.7 \pm 1.1$ seconds at baseline to $4.1 \pm 1.0$ seconds after 2 weeks, $5.2 \pm 1.5$ seconds after 6 weeks, and $6.5 \pm 1.5$ seconds after 12 weeks. Forty-five eyes (75.0\%) showed abnormal fluorescein staining of the cornea at baseline. The number of eyes with abnormal values decreased during the course of the study to $35(58.3 \%), 21$ (35.0\%), and seven eyes (11.7\%) at weeks 2,6 , and 12 , respectively (Table 2 ).

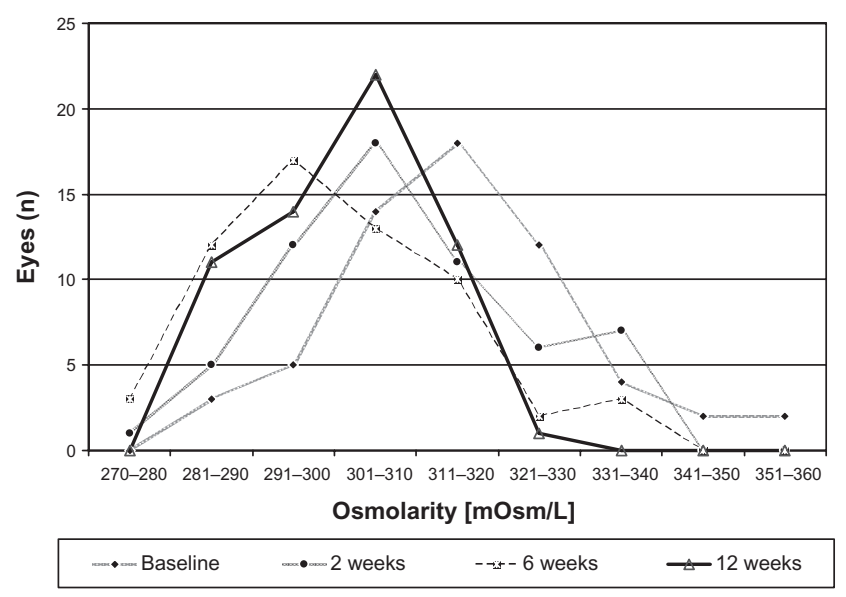

Figure I Distribution of osmolarities at latanoprost baseline and 2, 6, and I2 weeks after changing medication to preservative-free tafluprost. 


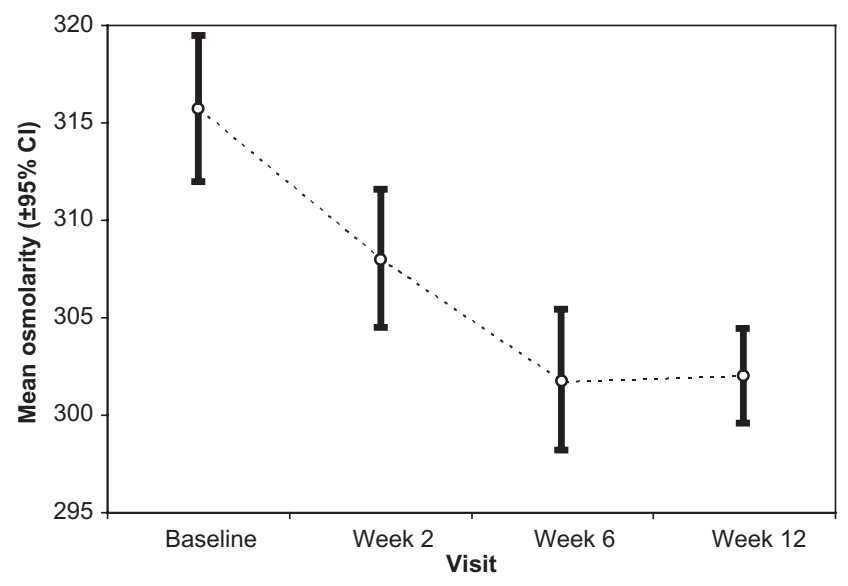

Figure 2 Mean osmolarity and 95\% confidential intervals $(\mathrm{Cl})$ at baseline (latanoprost) and 2, 6, and 12 weeks after changing medication to preservative-free tafluprost.

\section{Effect on IOP}

Mean IOP $( \pm \mathrm{SD})$ was $16.4 \pm 3.0 \mathrm{mmHg}$ at treated baseline. At baseline, difference in IOP values between right and left eyes was not statically significant. IOP remained unchanged at 2 weeks $(16.3 \pm 2.9 \mathrm{mmHg} ; P=0.651)$, 6 weeks $(16.2 \pm 2.7 \mathrm{mmHg} ; P=0.673)$, and 12 weeks $(16.3 \pm 2.3 \mathrm{mmHg} ; P=0.820)$ after changing medication from preserved latanoprost to preservative-free tafluprost (Figure 3).

\section{Subjective complaints}

Subjective complaints decreased with every follow-up visit of tafluprost treatment. At the final visit 12 weeks after changing medication to preservative-free tafluprost, only $11(36.7 \%)$ out of 30 patients at baseline were still expressing subjective complaints (Table 2).

\section{OSDI and OSSG questionnaires}

The OSDI questionnaire showed that during latanoprost baseline, 16 patients (53.3\%) had mild dry eye complaints, while after 12 weeks the number of patients with these symptoms was reduced to eight (26.7\%) (Figure 4). The OSSG questionnaire revealed that $40.0 \%$ of patients felt dry eye
Table I Patient demographics $(n=30)$

\begin{tabular}{ll}
\hline Mean age (years) & 64.2 \\
$\quad$ Range (years) & $35-79$ \\
SD & 13.9 \\
Sex & \\
Male & 26 \\
Female & 4 \\
Glaucoma since years (mean) & 3.6 \\
Range (years) & $0.5-15$ \\
\hline
\end{tabular}

Abbreviation: SD, standard deviation.

symptoms some of the time at baseline. Twelve weeks after changing medication to preservative-free tafluprost, these symptoms remained in only $26.7 \%$ of patients (Figure 5). Using the OSDI questionnaire, our study revealed that 16 patients $(53.3 \%)$ had mild complaints typical to dry eye syndrome, while 12 patients $(40.0 \%)$ had no complaints at all. Twelve weeks after changing medication to preservativefree tafluprost, the number of patients with mild complaints was reduced to eight (26.6\%). Nineteen patients $(63.3 \%)$ reported no complaints at all. The OSSG questionnaire showed that 12 patients $(40 \%)$ had dry eye symptoms some of the time and four (13.3\%) most of the time, while $40 \%$ had no complaints. After 12 weeks of tafluprost treatment, dry eye symptoms present "only some of the time" were noticed by eight patients $(26.7 \%)$, while the number of patients reporting dry eye symptoms present "most of the time" decreased to three $(10.0 \%)$. Consequently the number of patients reporting "no complaints" increased to 17 $(56.7 \%)$.

\section{Discussion}

Currently, the only proven and accepted method of preserving visual function in patients with glaucoma is to lower IOP. Although a variety of glaucoma genotypes and phenotypes exist, reduction of IOP remains the only modifiable risk factor delaying or preventing visual loss. ${ }^{1,20-22}$

Chronic use of most IOP-lowering medications is associated with various adverse reactions: allergies, conjunctivitis, contact dermatitis, punctate keratitis, or even failure of filtration surgery. ${ }^{23-25}$ This toxicity seems rather to be

Table 2 Changes of patient complaints, mean tear film break-up time ( $\pm S D)$ and abnormal corneal fluorescein staining at baseline (latanoprost), 2, 6, and 12 weeks after changing medication to preservative-free tafluprost

\begin{tabular}{llllll}
\hline & Baseline & Week 2 & Week 6 & Week I2 & $\begin{array}{l}\text { P-value (I2 weeks vs } \\
\text { baseline) }\end{array}$ \\
\hline Dry eye complaints $\mathrm{n}=30$ (patients) & $30(100 \%)$ & $19(63.3 \%)$ & II (36.7\%) & II (36.7\%) & $<0.05$ (McNemar) \\
Tear film break-up time (seconds) $\mathrm{n}=60$ (eyes) & $3.7 \pm 1.1$ & $4.1 \pm 1.0$ & $5.2 \pm 1.5$ & $6.5 \pm 1.5$ & $<0.00$ I (paired $t$-test) \\
$\begin{array}{l}\text { Abnormal fluorescein staining of the cornea } \\
\mathrm{n}=60 \text { (eyes) }\end{array}$ & $45(75.0 \%)$ & $35(58.3 \%)$ & $21(35 \%)$ & $7(12 \%)$ & $<0.005$ (McNemar) \\
\hline
\end{tabular}

Abbreviation: SD, standard deviation. 


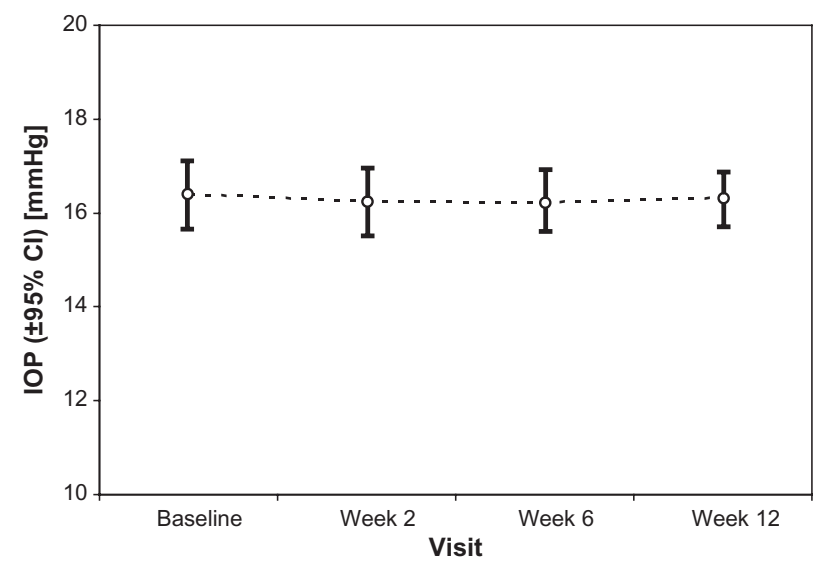

Figure 3 Mean intraocular pressure (IOP) and $95 \%$ confidential intervals $(\mathrm{Cl})$ at baseline (preserved latanoprost) and 2, 6, and 12 weeks after changing medication to preservative-free tafluprost.

associated with the preservative BAK than with the active component of the medication. ${ }^{26}$ From this point of view, preservative-free preparations in unit-dose presentation, are a viable alternative to traditional multidose bottles.

Our study found no statistically significant difference in the IOP levels of patients treated with the preservative-free prostaglandin analog tafluprost and the BAK-preserved formulation of the prostaglandin analog latanoprost. The average latanoprost baseline IOP $(16.4 \pm 2.9 \mathrm{mmHg})$ was similar to the average IOP after 3 months of preservative-free tafluprost therapy $(16.3 \pm 2.3 \mathrm{mmHg})$. Our results confirm the findings of Uusitalo and colleagues who found that IOP remained unchanged 12 weeks after changing medication from BAKpreserved latanoprost to preservative-free tafluprost. ${ }^{27}$ Miyashiro and colleagues found that changing medication from BAK-preserved latanoprost $0.005 \%$ monotherapy to a

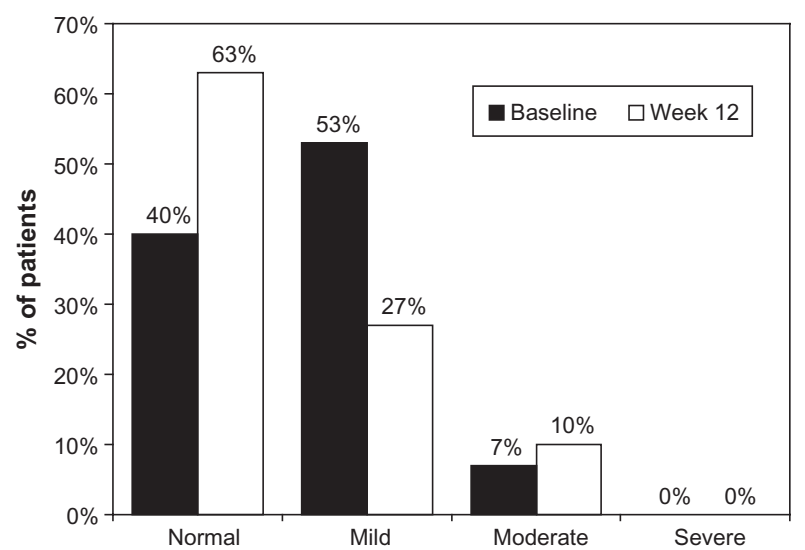

Figure 4 Severity of ocular symptoms at baseline (preserved latanoprost) and 12 weeks after changing medication to preservative-free tafluprost evaluated by using the OSDI questionnaire.

Abbreviation: OSDI, Ocular Surface Disease Index.

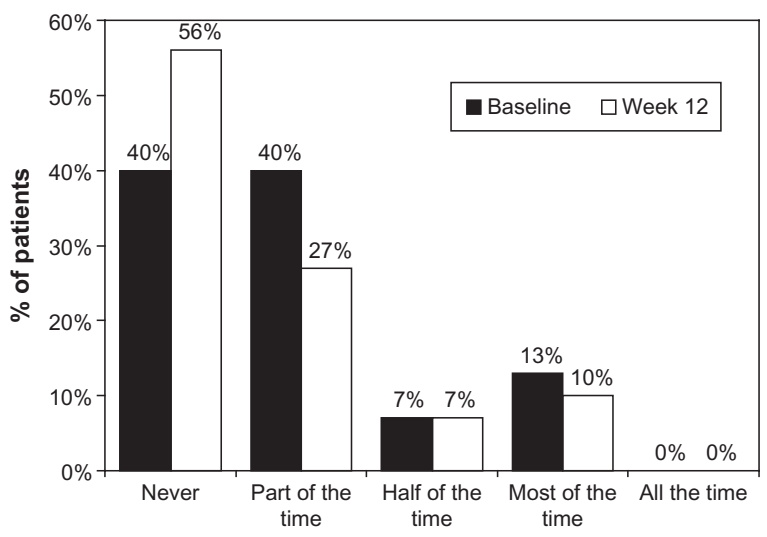

Figure 5 Frequency of ocular symptoms at baseline (preserved latanoprost) and 12 weeks after changing medication to preservative-free tafluprost evaluated by using the OSSG questionnaire.

Abbreviation: OSSG, Ocular Surface Symptoms in Glaucoma Scale.

sofZia ${ }^{\mathrm{TM}}$-(Alcon Inc, Fort Worth, TX) preserved formulation of travoprost $0.004 \%$ resulted in a similar IOP control. ${ }^{28}$ The findings from these studies show that IOP is not changed significantly after changing medication from BAK-containing to preservative-free or sofZia ${ }^{\mathrm{TM}}$-preserved prostaglandin analog formulations. Therefore BAK might not be necessary for these prostaglandin analogs to enhance their corneal penetration and to achieve better IOP control. ${ }^{29}$ This may be due to the lipophilic nature of prostaglandin analogs. Hamacher and colleagues demonstrated in a randomized investigator masked cross-over study that BAK as a preservative has no influence on the effectiveness of tafluprost. IOP was measured four times per day at 1 and 4 weeks of treatment. There was no statistically significant difference between BAK-preserved tafluprost and preservative-free tafluprost $(P=0.957) .{ }^{30}$

Several studies have revealed discrepancies between dry eye symptoms and clinical signs. Signs specific for a single subtype of dry eye demonstrated poor correlation to disease severity in milder patients. Tear film osmolarity was found to be the single best marker of disease severity across normal, mild or moderate, and severe dry eye disease categories. ${ }^{12}$ Our study aimed to investigate osmolarity levels after switching from a BAK-containing PGA formulation to preservative-free tafluprost. Mean tear osmolarity level with BAK-preserved latanoprost was $315 \mathrm{mOsm} / \mathrm{L}$. Two weeks after switching to preservative-free tafluprost, mean osmolarity decreased statistically significantly to $308 \mathrm{mOsm} / \mathrm{L}$, and to $302 \mathrm{mOsm} / \mathrm{L}$ after 12 weeks $(P<0.001 ; t$-test). According to Lemp and colleagues, the most sensitive threshold between normal and mild or moderate subjects was found to be $308 \mathrm{mOsm} / \mathrm{L}$. At a cut-off of $312 \mathrm{mOsm} / \mathrm{L}$, tear hyperosmolarity exhibited $73 \%$ sensitivity and $92 \%$ specificity. By contrast, the other 
common tests exhibited either poor sensitivity (corneal staining, 54\%; conjunctival staining, 60\%; meibomian gland grading, 61\%) or poor specificity (TBUT, 45\%; Schirmer test, 51\%). Differences in osmolarity were found to correlate with increasing disease severity $\left(r^{2}=0.32\right) .{ }^{31}$ Another study by Luo and colleagues proved that hyperosmolarity induces apoptosis of human corneal epithelial cells. ${ }^{32}$

Evaluating other common signs for ocular surface disease such as a decrease in TBUT, we found that TBUT increased statistically significantly from $3.7 \pm 1.1$ seconds at latanoprost baseline to $6.5 \pm 1.5$ seconds 12 weeks after changing medication to preservative-free tafluprost $(P<0.001)$. Abnormal corneal fluorescein staining was observed in $45(75.0 \%)$ eyes at baseline. Twelve weeks after the change of medication only seven $(11.7 \%)$ eyes showed abnormal values $(P<0.005)$. This improvement of signs of the ocular surface after changing medication from a BAK-preserved to a preservative-free prostaglandin analog medication confirm the findings of Uusitalo and colleagues. They showed that abnormal corneal fluorescein staining reduced statistically significantly from $81.6 \%$ of cases to $40.6 \%$. TBUT improved statistically significantly from $4.5 \pm 2.5$ seconds to $7.8 \pm 4.9$ seconds..$^{27}$

Leung and colleagues showed that $59 \%$ of patients with glaucoma or ocular hypertension have symptoms of ocular surface disease, with $27 \%$ being severe. ${ }^{33}$ The overall OSDI score is defined as normal ( $0-12$ points), mild (13-22 points), moderate (23-32 points), or severe (33-100 points) ocular surface disease. ${ }^{34}$ Severity of patient complaints with their medication decreased during the course of our study. Using the OSDI questionnaire the study revealed that $53.3 \%$ of patients had mild complaints typical for dry eye syndrome, while $40.0 \%$ had no complaints at all. Twelve weeks after changing the medication to preservative-free tafluprost, the number of patients with mild complaints was reduced from $53.3 \%$ at baseline to $26.6 \%$. Furthermore, $63.3 \%$ of patients reported no complaints at all. The OSSG questionnaire showed great improvement with respect to the frequency of ocular symptoms: 12 weeks after change of medication to preservative-free tafluprost, dry eye symptoms improved significantly $(P>0.005)$. Data from Uusitalo and colleagues revealed that during treatment with preservative-free tafluprost the number of patients having dry eye sensation decreased from $64.6 \%$ to $39.4 \% .{ }^{27}$ According to Schiffman and colleagues, the OSDI is a valid and reliable instrument for measuring the severity of dry eye disease, and it possesses psychometric properties that are needed as an end point in clinical trials. ${ }^{35}$ Fechtner and colleagues found a good correlation between OSDI symptoms and the number and duration of glaucoma treatments used.$^{36}$ Other clinical studies have demonstrated that the withdrawal of preservatives may reduce adverse effects associated with eye drops while preservative-free glaucoma treatments may have clinically relevant benefits for patients. ${ }^{37,38}$ The findings of the present study confirm the results from these prior studies.

\section{Conclusion}

Our results suggest that a change of medication from BAKpreserved latanoprost to preservative-free tafluprost is able to normalize tear osmolarity, to improve subjective symptoms, reduce corneal fluorescein staining, and improve TBUT while maintaining effective IOP control. These findings may be of specific importance for all glaucoma patients with sensitive and dry eyes.

\section{Acknowledgment/disclosure}

None of the authors has financial interest in Santen or any of the products mentioned in this study. Ingrida Janulevičiene is a speaker for Santen Oy. Friedemann Kimmich, PhD (eyecons) provided support in medical writing of the present study with financial support by Santen Oy.

\section{References}

1. AGIS Investigators. The Advanced Glaucoma Intervention Study (AGIS): 7. The relationship between control of intraocular pressure and visual field deterioration. Am J Ophthalmol. 2000;130:429-440.

2. Goldberg I, Clement CI, Chiang TH, et al. Assessing quality of life in patients with glaucoma using the Glaucoma Quality of Life-15 (GQL-15) questionnaire. J Glaucoma. 2009;18:6-12.

3. Buron N, Micheau O, Cathelin S, et al. Differential mechanisms of conjunctival cell death induction by ultraviolet irradiation and benzalkonium chloride. Invest Ophthalmol Vis Sci. 2006;47: 4221-4230.

4. Hopes M, Broadway D. Preservative-free treatment in glaucoma is a sensible and realistic aim for the future. European Ophthalmic Review. 2010;4:23-28.

5. Noecker R. Effects of common ophthalmic preservatives on ocular health. Adv Ther. 2001;18:205-215.

6. Lemp MA, Zimmerman LE. Toxic endothelial degeneration in ocular surface disease treated with topical medications containing benzalkonium chloride. Am J Ophthalmol. 1988;105(6):670-673.

7. Rieger G. The importance of the precorneal tear film for the quality of optical imaging. Br J Ophthalmol. 1992;76:157-158.

8. Asbell PA, Lemp MA. Dry Eye Disease: The Clinician's Guide to Diagnosis and Treatment. New York, NY: Thieme; 2006.

9. Widmaier EP, Raff H, Strang KT. Vander's Human Physiology. New York, NY: McGraw-Hill; 2008;11:108-120.

10. Schaeffer AJ. Osmotic pressure of the extraocular and intraocular fluids. Arch Opthalmol. 1950;43:1026-1035.

11. Tomlinson A. Tear film osmolarity: determination of a referent for dry eye diagnosis. Invest Ophthalmol Vis Sci. 2006;47:4309-4315.

12. Sullivan BD, Whitmer D, Nichols KK, et al. An objective approach to dry eye disease severity. Invest Ophthalmol Vis Sci. 2010;51:6125-6230.

13. Suzuki M, Massingale ML, Fen Ye F, et al. Tear osmolarity as a biomarker for dry eye disease severity. Invest Ophthalmol Vis Sci. 2010;51: $4557-4561$. 
14. Farris RL. Tear osmolarity: a new gold standard? Adv Exp Med Biol. 1994;350:495-503.

15. Mastmann GJ, Baldes EJ, Henderson JW. The total osmotic pressure of tears in normal and various pathologic conditions. Arch Ophthalmol. 1961;65:509-513.

16. Terry JE, Hill RM. Human tear osmotic pressure: diurnal variations and the closed eye. Arch Ophthalmol. 1978;96:120-122.

17. Liang H, Baudouin C, Pauly A, Brignole-Baudouin F. Conjunctival and corneal reactions in rabbits following short- and repeated exposure to preservative-free tafluprost, commercially available latanoprost and $0.02 \%$ benzalkonium chloride. $\mathrm{Br} J$ Ophthalmol. 2008;92:1275-1282.

18. Brasnu, E, Brignole-Baudouin, F, Riancho L, Guenoun JM, Warnet JM, Baudouin C. In vitro effects of preservative-free tafluprost and preserved latanoprost, travoprost, and bimatoprost in a conjunctival epithelial cell line. Curr Eye Res. 2008;33:303-312.

19. Lemp MA. Report of the National Eye Institute/Industry Workshop on Clinical Trials in Dry Eyes. CLAO J. 1995;21:221-232.

20. Drance S, Anderson DR, Schulzer M. Risk factors for progression of visual field abnormalities in normal-tension glaucoma. Am J Ophthalmol. 2001;131:699-708.

21. Leske MC, Heijl A, Hyman L, et al. Predictors of long-term progression in the early manifest glaucoma trial. Ophthalmology. 2007; 114:1965-1972.

22. Kass MA, Heuer DK, Higginbotham EJ, et al. The Ocular Hypertension Treatment Study. A randomized trial determines that topical ocular hypertensive medication delays or prevents the onset of primary openangle glaucoma. Arch Ophthalmol. 2002;120:701-713.

23. Lee DA, Higginbotham EJ. Glaucoma and its treatment: a review. Am J Health Syst Pharm. 2005;62:691-699.

24. Baudouin C. Allergic reaction to topical eyedrops. Curr Opin Allergy Clin Immunol. 2005;5:459-463.

25. Fisher AA. Allergic contact dermatitis and conjunctivitis from benzalkonium chloride. Cutis. 1987;39:381-383.

26. Whitson JT, Cavanagh HD, Lakshman N, Petroll WM. Assessment of corneal epithelial integrity after acute exposure to ocular hypotensive agents preserved with and without benzalkonium chloride. Adv Ther. 2006;23(5):663-671
27. Uusitalo H, Chen E, Pfeiffer N, et al. Switching from a preserved to a preservative-free prostaglandin preparation in topical glaucoma medication. Acta Ophthalmologica. 2010;88:329-336.

28. Miyashiro MJ, Lo SC, Stewart JA, et al. Efficacy, safety, and tolerability of travoprost $0.004 \%$ BAK-free versus prior treatment with latanoprost 0.005\% in Japanese patients. Clin Ophthalmol. 2010;4:1355-1359.

29. Lewis RA, Katz GJ, Weiss MJ, et al; Travoprost BAC-free Study Group. Travoprost $0.004 \%$ with and without benzalkonium chloride: a comparison of safety and efficacy. J Glaucoma. 2007;16:98-103.

30. Hamacher T, Airaksinen J, Saarela V, et al. Efficacy and safety levels of preserved and preservative-free tafluprost are equivalent in patients with glaucoma or ocular hypertension: results from a pharmacodynamics analysis. Acta Ophthalmol Suppl (Oxf). 2008;242:14-19.

31. Lemp MA, Bron AJ, Baudouin C, et al. Tear osmolarity in the diagnosis and management of dry eye disease. Am J Ophthalmol. 2011;151: 792-798.

32. Luo L, Li DQ, Pflugfelder SC. Hyperosmolarity-induced apoptosis in human corneal epithelial cells is mediated by cytochrome $\mathrm{c}$ and MAPK pathways. Cornea. 2007;26:452-460.

33. Leung EW, Medeiros FA, Weinreb RN. Prevalence of ocular surface disease in glaucoma patients. J Glaucoma. 2008;17:350-355.

34. Miller KL, Walt JG, Mink DR, et al. Minimal clinically important difference for the ocular surface disease index. Arch Ophthalmol. 2010; 128:94-101.

35. Schiffman R, Christianson MD, Jacobsen G, Hirsch JD, Reis BL. Reliability and validity of the ocular surface disease index. Arch Ophthalmol. 2000;118:615-621.

36. Fechtner RD, Godfrey DG, Budenz D, Stewart JA, Stewart WC, Jasek MC. Prevalence of ocular surface complaints in patients with glaucoma using topical intraocular pressure-lowering medications. Cornea. 2010;29:618-621.

37. Baudouin C. Detrimental effect of preservatives in eyedrops: implications for the treatment of glaucoma. Acta Ophthalmol. 2008;86: 716-726.

38. Pisella PJ, Pouliquen P, Baudouin C. Prevalence of ocular symptoms and signs with preserved and preservative free glaucoma medication. Br J Ophthalmol. 2002;86:418-423.
Clinical Ophthalmology

\section{Publish your work in this journal}

Clinical Ophthalmology is an international, peer-reviewed journal covering all subspecialties within ophthalmology. Key topics include: Optometry; Visual science; Pharmacology and drug therapy in eye diseases; Basic Sciences; Primary and Secondary eye care; Patient Safety and Quality of Care Improvements. This journal is indexed on

\section{Dovepress}

PubMed Central and CAS, and is the official journal of The Society of Clinical Ophthalmology (SCO). The manuscript management system is completely online and includes a very quick and fair peer-review system, which is all easy to use. Visit http://www.dovepress.com/ testimonials.php to read real quotes from published authors. 